\title{
DAS DILEMMA DER LIEBE UND DIE POETIK DER AUFRICHTIGKEIT. HEINRICH HEINE UND ALAIN BADIOU
}

ÓSCAR PALACIOS BUSTAMANTE

\author{
NEO \\ I just have never ... \\ RAMA-KANDRA \\ ... heard a program speak of love? \\ NeO \\ It's a ... human emotion. \\ RAMA-KANDRA
}

No, it is a word. What matters is the connection the word implies.

The Matrix. Revolutions (2003)

\begin{abstract}
After shortly outlining what can be called hermeneutics of love, the author carries out a brief interpretation of a poem written by Heinrich Heine from the perspective of Alain Badiou's theory of love. For this purpose, and after analyzing the structure and content of Heine's poem itself, the author synthetizes and formalizes four different conceptualizations of love as explained by Badiou in a lecture from 2006 (Mexico City). The author finally uses the conceptual background of Badiou's proposal to re-interpret Heine's poem and to show that Heine's poem offers another key concept missing in Badiou's theory for the understanding of the experience of love, i. e. the concept of sincerity (Aufrichtigkeit).
\end{abstract} Creative Commons Attribution License (http://creativecommons.org/licenses/by/4.0). 


\section{Einführung}

Was ist Liebe? Wann entstand die Liebe? Ist die Liebe eine intersubjektive Konstruktion, die eine entscheidende Rolle für das Gesellschaftliche spielt, oder handelt es sich nicht vielmehr um eine psychologische Erfahrung, die auf eine bloß eigentümliche Sphäre beschränkt bliebt? Scheint es grundsätzlich möglich, eine allgemeine Form der Liebe vorzuschlagen, gerade in Anbetracht der unzählbaren Geschichten, überlieferten Erfahrungen und Werke, die, räumlich und zeitlich so ungemein weitgestreut, von der Liebe handeln? Wie kann Liebe überhaupt in unserer Zeit theoretisiert werden?

Das, was wir heutzutage unter „Liebe“ verstehen, war nicht immer dieserart da, d. h.: die Liebe als Kennzeichen oder sogar Begriff einer bestimmten Erfahrung oder einer Menge von Erfahrungen ist das Ergebnis einer geschichtlichen Entwicklung. Die Liebe hat ihre eigene Geschichte, die vieldeutig und vielfältig wie jedes geschichtliche Phänomen sein kann. Darum würde eine Hermeneutik der Liebe vom Standpunkt ausgehen, dass es in jeder Zeit und jedem Lebensraum eine Erfahrung der Liebe gibt, deren Namen, Gestalten und mögliche Zusammenhänge spezifisch sind, d. h., dass jene Namen, Gestalten und Zusammenhänge nicht direkt gegen diejenigen einer anderen Zeit und eines anderen Lebensraumes ausgetauscht werden können. Wenn ein solcher hermeneutischer Austausch stattfinden könnte, wie wäre dieser philosophisch zu vollziehen?

Im Rahmen der uns bekannten Philosophie und - um das Wort Hans Blumenbergs zu verwenden - Protophilosophie, ${ }^{1}$ die als der Anfang der abendländischen Wissenschaft überhaupt bezeichnet werden kann, finden wir schon Begriffe und sogar Theorien der Liebe und der Sexualität. Die ontologisch-kosmologischen Termini der Liebe (Philótes, Philíe, Aphrodíte ) bei Empedokles von Akragas ${ }^{2}$ oder die Beschreibung des Geschlechtsverkehres (xynousíe ) bei Demokrit von Abdera ${ }^{3}$ spiegeln beispielsweise die Tatsache wider, dass schon am Anfang der Philosophie verschiedene Gedanken und Überlegungen über die Erfahrung der Liebe im Kern unterschiedlicher Darstellungen und verschiedenartiger Untersuchungen stehen.

1 Vgl. Blumenberg Hans, „Der Sturz des Protophilosophen. Zur Komik der reinen Theorie - anhand einer Rezeptionsgeschichte der Thales-Anekdote" in Preisendanz W. und Warning R. (Hrsg.), Das Komische, München, Fink, Poetik und Hermeneutik 7, 1976, S. 11-64; vgl. auch Blumenberg Hans, Das Lachen der Thrakerin. Eine Urgeschichte der Theorie, Frankfurt am Main, Suhrkamp, 1987, sowie Blumenberg Hans, Theorie der Unbegrifflichkeit, Frankfurt am Main, Suhrkamp, 2007, S. $16 \mathrm{ff}$.

2 Vgl. beispielsweise Diels Hermann und Kranz Walther, Die Fragmente der Vorsokratiker, Berlin, Weidmann, 1956, Kapitel 31, B 17-19 (fortan: „DK 31 B 17-19“).

3 Vgl. DK 68 B 32. 
Man könnte sogar behaupten, dass bestimmte Ontologien und Anthropologien der Liebe und der Sexualität ursprünglich zur Philosophie gehören. Paradigmatisch ist natürlich in diesem Sinne Platos Dialog Symposion, in dem diese langen und bunten Denktraditionen, Quellen und Betrachtungen über die Liebe gewissermaßen geordnet werden, um sich zu einem bestimmten Mosaik zu fügen, auf dem Plato selbst eine eigene und komplexe Theorie der Liebe aufgebaut und entwickelt hat, ein Theorie, wohlgemerkt, die sich nachträglich als entscheidend für die abendländische, sowohl vorchristliche als auch christliche, Tradition erweisen würde.

Im Rahmen der Gegenwartsphilosophie, d. h. nach der Entstehung, Entwicklung und den sogenannten Krisen des neuzeitlichen Subjektes hat der marokkanisch-französische Philosoph Alain Badiou (1937-) diese Verbindung von Liebe, Sexualität und Philosophie erneut intensiv bearbeitet. ${ }^{4}$ Badiou zufolge stelle eine solche Bearbeitung eine platonische Geste dar, die nach dem sogenannten Tod Gottes zu einem „Platonismus des Vielfachen“" gehöre, ${ }^{5}$ in dem Sinn, dass die Liebe eine Bedingung der Philosophie selbst sei. Unsere zeitgenössischen Erfahrungen und Gedanken über die Liebe und die Sexualität konfigurieren solcherart eine Wahrheitsprozedur, auf deren Anwesenheit und Seinsweise die Philosophie basieren könne, um sich zu erneuern.

Das Anliegen des vorliegenden Aufsatzes ist es, den Versuch zu unternehmen, einige Betrachtungen Badious über die Liebe mit einem kurzen Liebesgedicht aus der Spätromantik beziehungsweise der sogenannten Kritik der Romantik hermeneutisch-philosophisch in Verbindung zu bringen, nämlich mit einem Gedicht Heinrich Heines (1797-1856), das „Emma“ gewidmet ist, und das vielleicht zwischen 1824 und 1836 entstand. ${ }^{6}$

4 Vgl. beispielsweise Badiou Alain und Truong Nicolas, Eloge de lamour, Paris, Flammarion, 2009; Badiou Alain, Le Séminaire. Théorie du mal, théorie de lamour, 1990-1991, Paris, Fayard, 2018; Badiou Alain und Cassin Barbara, Il n'y a pas de rapport sexuel. Deux leçons sur "L'Etourdit " de Lacan, Paris, Fayard, 2010, unter anderen Publikationen, Vorträge und Interviews, die sich ausführlich dieser Problematik widmen.

5 Badiou beschreibt und demonstriert für den Platonismus des Vielfachen mit den folgenden Worten: "Ce qu'un philosophe moderne retient de la grande sophistique est le point suivant : lêtre est essentiellement multiple. Déjà Platon, dans Le Théétète, pointait que l'ontologie sous-jacente à la proposition sophistique tenait dans la mobilité multiple de lêtre, et, à tort ou à raison, il couvrait cette ontologie du nom de Héraclite. Mais Platon réservait les droits de l'Un. Notre situation est plus complexe, car nous avons à prendre acte, à l'école de la grande sophistique moderne, qu'après de durs avatars, notre siècle aura été celui de la contestation de l'Un. Le sans-être de l'Un, l'autorité sans limite du multiple, nous ne pouvons revenir là-dessus. Dieu est réellement mort, et toutes les catégories qui en dépendaient dans l'ordre de la pensée de lêtre. La passe qui est la nôtre est celle d'un platonisme du multiple." (Badiou Alain, Manifeste pour la philosophie, Paris, Seuil, 1989, S. 85).

6 Wir folgen die Ausgabe und Notizen von Klaus Briegleb. Vgl. Heine Heinrich, Sämtliche Gedichte in zeitlicher Folge, Herausgegeben von Klaus Briegleb, Frankfurt am Main und Leipzig, Insel Verlag, 
Warum Heine mit Badiou in Verbindung bringen?

Berühmt ist Heines Kritik, die er an der Romantik übte. ${ }^{7}$ Sein Werk stellt sich ausdrücklich als das Bewusstsein der Wende des 18. Jahrhunderts, d. h. eines Wandels von literarischen Paradigmen, vor. ${ }^{8}$ Unsere hermeneutische Voraussetzung diesbezüglich ist, dass bei der Poesie Heines auch unterschiedliche Liebesparadigmen zu finden sind, d. h., dass die Poesie Heines der selbstbewusste Treffpunkt verschiedener Formen und Ausdrücke der Liebe ist, wobei der Dichter mit denselben abwechselnd und in verschiedenen Tönen kreativ, kritisch und sogar manchmal ironisch spielt. ${ }^{9}$

Wie wir sehen werden, ist dieses Bewusstsein des Wandels von Liebesparadigmen ein Schluss für die philosophisch-geschichtliche Auslegung der Liebe Badious. Das Ziel und die Herausforderung unseres Versuches ist dann, uns der von Heine dichterisch gestellten Problematik der Liebe mit Hilfe der philosophischen Theorie der Liebe Badious anzunähern, wobei es möglich sein wird, solche Liebesparadigmen als intersubjektive und gesellschaftliche Erfahrungen der Moderne und Gegenwart zu unterscheiden. Daraus ergeben sich des Weiteren die Teile und die Methode dieses Aufsatzes: im ersten Teil werden wir das oben erwähnte Gedicht Heines in seiner Form und Inhalt analysieren, um ein bestimmtes Problem auszulegen, das im zweiten Teil aus einer badiouschen Perspektive bearbeitet wird. Durch diesen hermeneutisch-philosophischen Austausch wird deutlich gemacht werden, inwieweit das Gedicht Heines eine intersubjektive Erfahrung der Aufrichtigkeit gegenüber der Liebe ausgedrückt wird, die mit Hilfe des begrifflichen Rahmens von Badiou formalisiert werden kann.

Insel Taschenbuch, 1997, S. 397. Vgl. auch Heine Heinrich, Verschiedene in Düsseldorfer Heine-Ausgabe (DHA), Bd. 2, Herausgegeben von Manfred Windfuhr, Hamburg, Hoffmann und Campe, 1997, S. 51f.

7 Vgl. Heine H., Die romantische Schule in DHA, Bd. 8/1, S. 121-252.

8 Heine notiert: „Um meine Wiege spielten die letzten Mondlichter des 18ten und das erste Morgenroth des 19ten Jahrhunderts." (Heine H., Prosanotizen in DHA, Bd. 10, S. 339).

9 Vgl. beispielsweise das berühmte kurze Gedicht: „Das Fräulein stand am Meere / Und seufzte lang und bang, / Es rührte sie so sehre / Der Sonnenuntergang. // Mein Fräulein! seyn Sie munter, / Das ist ein altes Stück; / Hier vorne geht sie unter / Und kehrt von hinten zurück." (Heine Heinrich, Verschiedene in DHA, Bd. 2, S. 35f). 


\section{Das Gedicht von Heinrich Heine}

Lesen wir zuerst das Gedicht von Heine durch:

Emma, sage mir die Wahrheit:

Ward ich närrisch durch die Liebe?

Oder ist die Liebe selber

Nur die Folge meiner Narrheit?

Ach! mich quälet, teure Emma, Außer meiner tollen Liebe, Außer meiner Liebestollheit, Obendrein noch dies Dilemma.

Nummerieren wir jetzt die Verszeilen:

1. Emma, sage mir die Wahrheit:

2. Ward ich närrisch durch die Liebe?

3. Oder ist die Liebe selber

4. Nur die Folge meiner Narrheit?

5. Ach! mich quälet, teure Emma,

6. Außer meiner tollen Liebe,

7. Außer meiner Liebestollheit,

8. Obendrein noch dies Dilemma.

Das Gedicht besteht aus zwei vierzeiligen Strophen mit trochäischen Vierhebern. Die Kadenz ist in jedem Vers weiblich oder klingend und das Reimschema ist: [ABCA DBED]. So kann dann der Aufbau des Gedichtes schematisiert werden (wo die betonten Silben als „-“ und die unbetonten Silben als „U“ dargestellt sind):

$$
\begin{aligned}
& \text { 1. - U - U - U - U (A) } \\
& \text { 2. - U - U - U - U (B) } \\
& \text { 3. - U - U - U - U (C) } \\
& \text { 4. - U - U - U - U (A) } \\
& \text { 5. - U - U - U - U (D) } \\
& \text { 6. - U - U - U - U (B) } \\
& \text { 7. - U - U - U - U (E) } \\
& \text { 8. - U - U - U - U (D) }
\end{aligned}
$$


Das Gedicht handelt von einer Problemstellung, die in der Form einer Bitte (1.), einer Fragestellung (2.-4.) und einer Wehklage (5.-8.) ausgedrückt wird. Worin besteht das Problem, das vom Dichter hier gestellt wird?

Zuallererst richtet sich das lyrische Ich an die Empfängerin der Bitte: „Emma“. Diese Stimme führt dann den Grund oder den Kern des Problems ein, nämlich eine Wahrheit. Das Problem des lyrischen Ich besteht in der Suche nach einer Wahrheit, die Emma und das lyrischen Ich in Verbindung bringt. Was für eine Verbindung steht auf dem Spiel? Durch eine doppelte Fragestellung unterscheidet die Stimme des Ich zwei mögliche Verbindungen zwischen sich und „Emma“: Entweder hat er sich zuerst in „Emma“ verliebt, um dadurch später „närrisch“ zu werden, oder er hat diese Liebe als eine bloße „Folge“ seiner schon früher vorhandenen „Narrheit“ erfahren. Die Form und Art oder sogar die Seinsweise der gesuchten Wahrheit ist von dieser zwischen dem lyrischen Ich und „Emma“ geteilten Entscheidung abhängig, die wir so formalisieren können:

$$
\text { Wahrheit(?) } \rightarrow((\text { Liebe } \rightarrow \text { Narrheit }) \text { v (Narrheit } \rightarrow \text { Liebe }))
$$

Erwähnenswert sind auch die Verben, die bei jeder Möglichkeit der Entscheidung verwendet werden: die erste Möglichkeit (Liebe $\rightarrow$ Narrheit) hat mit einem „Werden“ d. h. mit einem besonderen Vorgang zu tun, während die zweite Möglichkeit (Narrheit $\rightarrow$ Liebe) durch das Verb „sein“ ausgedrückt wird, d. h. diese zweite Möglichkeit hat mit der Idee einer Kontinuität von etwas schon Vorhandenem, von Gleichem, zu tun. Diesen zwei Möglichkeiten entsprechen in diesem Sinne zwei Modalitäten: entweder die Verwandlung eines Vorganges („Werden“) oder die Kontinuität des vorhandenen Gleichen („Sein“):

Wahrheit(?) $\rightarrow$ Werden v Sein

Auf jeden Fall stehen aber beide Möglichkeiten in Verbindung sowohl mit dem lyrischen Ich als auch mit „Emma“, daher schließt die Stimme des Ich das Gedicht mit einer Wehklage, die nochmals an „Emma“ gerichtet ist, und die den Ton der Entscheidung etabliert: dieses Problem, diese Suche nach einer Wahrheit oder „das Dilemma“, das darin besteht, diese Entscheidung zu treffen, wird vom lyrischen Ich als eine „Qual“ erfahren.

Wir können auch die Form hervorheben, in der diese Verwickelung in Verse gebracht wird: nachdem die Komponenten des Problems und die Verwickelten („Emma“ und das lyrische Ich) in der ersten Strophe präsentiert wurden, beginnt 
die zweite Strophe (5.) mit einer Hebung oder einer betonten Silbe, die mit dem leidenden Ausruf des Ich zusammenfällt. Darüber hinaus ist in derselben Strophe (7.) das einzige Kompositum des Gedichtes zu finden („Liebestollheit“), dessen Vers entscheidend das Reimschema definiert (7.... (E), anstatt der Wiederholung: 3./7... (B)). Dieses Kompositum setzt eigentlich in ein und demselben Substantiv die zwei oben erwähnten Termini („Liebe“ und „Narrheit“/,Tollheit“) zusammen, deren unklarer, problematischer Zusammenhang das Thema des Gedichtes selbst ist. Das Gedicht stellt die Frage nach dem Grund und der Art dieses Kompositums. Neben der Alliteration von Substantiven und Adjektiven („närrisch“/„Narrheit“, „toll“/,Tollheit“), können wir außerdem die Verwendung von „Liebe“ und „Emma“ erwähnen: „Liebe“ steht im Gedicht als Versschluss des Reims 2./6.... (B) und zugleich wird in den Verslinien 3./7. erwähnt, deren Reim ((C), (E)) nicht passt und somit die Ambiguität der Entscheidung hervorhebt. Zum Schluss kommt natürlich die Benennung des Problems, die den Vornamen der vom lyrischen Ich Geliebten enthält: „das Dilemma“. „Oben-da-rein“ ist „das Dil-Emma“. So verbindet sich das Ende des Gedichtes mit seinem Anfang, um sein Thema mit einer letzten Alliteration zusammenzufassen: „Emma...“, „...teure Emma, ...“ „...dies Dilemma“.

\section{Die badiousche Interpretation des Gedichtes}

In einem im Jahr 2006 in Mexiko-Stadt gehaltenen Vortrag ${ }^{10}$ unterscheidet Alain Badiou drei Hauptkonzeptionen oder Theorien der Liebe in der Moderne. Badiou zufolge ist das diesen drei Theorien Gemeinsame und zugleich der Ausgangspunkt oder die Hauptvoraussetzung seiner eigenen Theorie der Liebe, dass die Liebe eine Erfahrung „der Zwei“ ist, d.h., dass die Liebe keine Erfahrung des Anderen überhaupt ist, sondern die fokussiertere Erfahrung des Wissens, was es heißt, zwei zu sein. ${ }^{11}$

Mit anderen Worten: wenn ich (eine Eins, 1) mich in jemanden (eine andere Eins, 1) verliebe, liebe ich nicht die Alterität im allgemeinen Sinne, sondern die spezifische Alterität oder die Differenz zwischen mir und der anderen Person. Die Erfahrung dieser Differenz konstituiert dann den Übergang von der eigenen Eins-Erfahrung zur geteilten Zwei-Erfahrung. Aber was und auf welche Weise

10 Vgl. Badiou Alain, „Amor y psicoanálisis“ in Badiou Alain, El balcón del presente. Conferencias y entrevistas, Herausgegeben und übersetzt von Susana Bercovich, Françoise Ben Kemoun und Raúl Reyes Camargo, Mexiko-Stadt, Siglo XXI - Universidad Nacional Autónoma de México, 2008. S. $102-116$.

11 Vgl. Ibid., S. 103. 
wird genauer aus dieser Perspektive der Zwei erfahren? Badiou zufolge kann die Antwort in der Neuzeit dreifach sein.

Die erste Antwort oder die erste mögliche neuzeitliche Theorie der Liebe ist "die pessimistische Theorie", die darin besteht, dass trotz der Liebeserfahrung beide Eins getrennt bleiben, d. h., dass es keine echte Beziehung oder Verbindung zwischen den Geliebten gibt, sondern nur die Fiktion einer Beziehung, wobei einfach eine Einsamkeit neben einer anderen steht. Badiou zufolge kann diese pessimistische Theorie bei den französischsprachigen Moralisten des 17. Jahrhunderts gefunden werden, die die Kennzeichnung „Liebe“ eigentlich für das Spiel des Erotischen verwendet haben. Dies bedeutet, dass dieser Theorie zufolge die Liebe ein Betrug, eine Täuschung und Enttäuschung ist, während der Geschlechtsverkehr das einzig Reale ist. ${ }^{12}$ Wir können die Termini und den Übergang dieser pessimistischen Theorie so formalisieren:

\section{$1 \rightarrow 1,1$}

Die zweite von Badiou erwähnte Theorie ist die romantische Theorie: die Zwei wird eine neue Einheit, d. h. die Liebe ist dieser Theorie zufolge die eventuelle Erfahrung der Auflösung der Differenz zwischen beiden Eins. Die Liebe als romantische Fusion heißt dann, eine neue Eins - im wörtlichen Sinne - mit Leib und Seele $\mathrm{zu}$ werden, aber diese neue Eins kann nur durch eine dramatische Fusionierung entstehen, gerade weil diese neue Eins keinen Platz auf einer Welt finden kann, wo es nur einfache, einzelne, nicht fusionierte Eins-Einheiten gibt. ${ }^{13}$

Diese von der Welt getrennte „Supereins“ kann am Ende des Tages nur ihren dramatischen Tod erwarten, um vielleicht der Endlichkeit jeder Eins einfach eine andere Tonalität und Sphäre zu geben. Diese romantische Konzeption der Liebe kann so formalisiert werden:

$$
1 \rightarrow 1,1 \rightarrow \underline{1}
$$

Die dritte Theorie ist die Theorie der Liebe als Familientheorie: das Ziel und der Grund der Liebe ist die Entstehung und das Fortbestehen der Familie. Das Schicksal oder die Wahrheit der Liebe befindet sich dieser Theorie zufolge im Übergang von der Zwei der Geliebten zur Drei der Entstehung des Kindes (oder noch zu mehreren Eins). ${ }^{14}$ Dies kann wie folgt formalisiert werden:

12 Vgl. Ibidem.

13 Vgl. Ibid., S. 103ff.

14 Vgl. Ibid., S. 104. 
Bei diesen drei traditionellen Theorien oder Konzeptionen der Liebe steht diese auch in unterschiedlichen Zusammenhängen mit dem Gesellschaftlichen. Die pessimistische Theorie der Liebe setzt die Liebe einfach als eine gesellschaftliche Beziehung, gerade weil die erotische Beziehung eine mögliche Beziehung unter anderen ist. In diesem Sinne gehört die pessimistische Liebe „problemlos“ zum Gesellschaftlichen. Im Gegenteil dazu stellt die romantische Liebe eine Ausnahme der Gesellschaft dar, weil sie die Ausnahme jeder gesellschaftlichen Beziehung par excellence ist. Aus dieser Perspektive sagt man häufig, dass die Geliebten „allein auf der Welt" seien, wodurch sie sogar eine Art Kritik der gesellschaftlichen Welt selbst werden können. Und die Liebe als die Entstehung und das Fortbestehen des menschlichen Tieres, d. h. als Familienreproduktion wird als ein Sozialmittel, als ein Instrument des Gesellschaftlichen betrachtet. ${ }^{15}$

Entscheidend für unsere zeitgenössischen neuen Konzeptionen der Liebe ist Badiou zufolge die Kritik der Liebe, die die Psychoanalyse besonders bei Jacques Lacan (1901-1981) unternimmt. In diesem Kontext interpretiert Badiou im ersten Moment die psychoanalytische Kritik der Liebe als die tiefste und interessanteste Kritik der romantischen Liebe unserer Zeit, denn sie zeigt, dass es keine Liebesfusion geben kann, und dass die Differenz vielmehr immer auf dem Spiel steht. Aus dieser Perspektive wäre dann die Liebe eine Art Fiktion, die jene unmögliche Fusionsbeziehung ersetzen würde. Das Reale ${ }^{16}$ der Liebe wäre dann diese Unmöglichkeit, während die Liebe selbst zum Bereich des Imaginären gehörte und eigentlich von einer Art Wahnsinn oder Neurose kaum zu unterscheiden wäre. ${ }^{17}$

Darum interpretiert Badiou in einem zweiten Moment diese psychoanalytische Kritik der Liebe auch als eine Gefahr, denn die psychoanalytische Konzeption der Liebe würde de facto einen der pessimistischen Theorie der Liebe ähnlichen Skeptizismus darstellen. ${ }^{18}$ Gerade gegen diesen Skeptizismus und ohne zur roman-

15 Vgl. Ibidem.

16 Wir verwenden hier die konventionelle deutsche Übersetzung vom Terminus Lacans „le réel“, es steht aber zur Debatte, ob diese die passende Übersetzung ist, wobei die Termini Lacans mit jenen von der deutschsprachigen Transzendentalphilosophie verglichen werden sollten, damit „le réel“ als „das Wirkliche“ vielleicht besser ins Deutsche übersetzt werden könnte. Ich bedanke mich bei Herrn Philipp Nolz für diese Anmerkung.

17 Vgl. Ibid., S. 104f. Vgl. auch diesbezüglich Badiou A., L'Immanence des vérités. L'Être et lévénement 3, Paris, Fayard, 2018. Section IX: œuvres selon le devenir: amour, politique, Chapitre C25: l'œuvre d'amour: la scène du Deux, S. 611-624.

18 Vgl. Badiou A., „Amor y psicoanálisis“, art. cit., S. 105. 
tischen Liebe zurückzukehren schlägt Badiou eine vierte Theorie der Liebe vor, nämlich die Theorie der Liebe als ereignishaftes Subjekt.

Mit Hilfe der oben erwähnten Elemente können wir diese Theorie der Liebe Badious so zusammenfassen: jede Liebe beginnt mit einer Begegnung (rencontre), mit einer Begegnung einer Eins mit einer anderen Eins, die immer unerwartet und zufällig ist. Aber neben ihrem aleatorischen Charakter besteht das Besondere der Liebesbegegnung darin, dass nach der Begegnung der Eins mit der anderen Eins etwas - im strengen Sinne - absolut Neues geschehen ist, und dies bedeutet, dass die Eins nicht mehr nur (eine bloße) Eins sein kann. Etwas an der Eins will mehr sein, etwas an der Eins will über ihre eigene Einheit hinausgehen. Dies ist der ereignishafte Charakter der Liebesbegegnung, nämlich dass die Eins die Möglichkeit erfahren hat, nicht mehr Eins, sondern Zwei zu sein. Das Ereignis der Liebe besteht im Übergang von zwei passiven Eins zur kreativen Erfahrung der Zwei. Beide Eins können nicht mehr allein sein, weil sie nach der Begegnung erfahren haben, was es bedeutet, alles aus der Perspektive der Zwei zu erfahren, was noch etwas Mehreres als ein gewöhnliches Nebeneinanderstehen ist. Dies bedeutet aber nicht, dass die Zwei eine Fusion beider Eins ist. Die Kreation des Liebesereignisses ist keine über die Welt hinausgehende Supereins, sondern die Eröffnung einer weltlichen Perspektive, die aus beiden Eins komponiert wird. In diesem Sinne spricht Badiou von einer „Bühne der Zwei“ (la scène du Deux), auf der die Welt oder die Situation aus der Perspektive der spezifischen Differenz zwischen beiden Eins erfahren wird. Badiou stimmt Lacan darin zu, dass die Differenz immer bleibt, d. h., dass es weder eine Auflösung der Differenz noch eine Fusion gibt, aber da, wo Lacan den Ursprung einer auf Unmöglichkeit beruhenden Fiktion findet, formalisiert Badiou das Geschehen einer Quelle von neuen Möglichkeiten, ${ }^{19}$ oder mit den Worten von Byungchul Han: ein Wesen von Negativität:

Sie [die Bühne der Zwei] unterbricht die Perspektive des Einen und lässt die Welt aus dem Gesichtspunkt des Anderen oder des Unterschiedes neu erstehen. Die Negativität einer Umwälzung zeichnet die Liebe als Erfahrung und Begegnung aus [...] Es [das Ereignis] lässt etwas geschehen, worüber die Situation nicht Rechenschaft geben kann. Es unterbricht das Gleiche zugunsten des Anderen. Das Wesen des Ereignisses ist die Negativität des Bruchs, die etwas ganz Anderes beginnen lässt. ${ }^{20}$

19 Vgl. Ibid., 106ff. Vgl. auch Badiou A., Le Séminaire. Théorie du mal, théorie de l'amour, 1990-1991, S. 133-173.

20 Han Byungchul, Agonie des Eros, Berlin, Matthes \& Seitz Berlin, 2015, S. 57f. Ausdrücke in den Klammern wurden vom Verfasser eingefügt. Bei anderer Gelegenheit sollte diskutiert werden, was für eine Negativität das Ereignis bei Badiou genauer bedeuten kann, ein Zusammenhang, der leider in Hans Text unklar bleibt. 
Die ereignishafte Begegnung der Liebe sollte dann als eine neue Ziffer dargestellt werden:

$$
1 \rightarrow 2
$$

Und so können wir die weltliche Perspektive der Bühne der Zwei formalisieren:

$$
\frac{1 \rightarrow 2}{\text { Welt }}
$$

Aber Badiou zufolge besteht die Liebe nicht nur aus einer ereignishaften Begegnung: das Ereignis fordert auch einen Namen und einen Weg. Damit die Bühne der Zwei sich fortsetzen kann (sonst würde sie sich in ihrem Sein einfach auf das augenblickliche Auftauchen des Ereignisses beschränken), fordert sie früher oder später den Moment und die Wörter einer Aussage, nämlich beispielsweise den Moment, in dem man sagt: „Ich liebe dich“. Die ereignishafte Liebe fordert eine Benennung, die auch unendlich variieren kann. Aber worauf beruhen solche Variationen? Wodurch und wie setzt sich die ereignishafte Liebe fort? Nur auf der Welt selbst kann die ereignishafte Liebe sich fortsetzen und mit der Zeit ihrer Variationen fortbestehen. In diesem Sinne wird die Welt von der ereignishaften Liebe gezwungen, sich nach solchen Variationen zu rekonfigurieren, während die Folgen jener weltlichen Rekonfiguration auch verursachen, dass die (Komposition der) Zwei sich ebenso verändert. Nach dem Erkennen der immer anwesenden spezifischen Differenz in der ereignishaften Begegnung kann dann eine Benennung kommen, die einen neuen Weg, eine neue Seinsweise beider Eins, nämlich die Bühne der Zwei, und einen neuen Bund zwischen der Welt und beiden Eins eröffnet. In diesem Sinne sagt Badiou, dass die Liebe ein selbstständiger Operator der Welt (anstatt eines Terminus jener Welt) ist. ${ }^{21}$ So wird dann die ereignishafte Liebe in der Form der Bühne der Zwei eine Art von subjektivem „Prisma“, durch dessen Spiel von Licht und Schatten das Unendliche auf der Welt erfahren werden kann:

$2 \rightarrow$ Welt $\rightarrow 2^{\prime}$

$2^{\prime} \rightarrow$ Welt' $\rightarrow 2^{\prime \prime}$

$2^{\prime \prime} \rightarrow$ Welt" $\rightarrow 2^{\prime \prime \prime}$

... ad infinitum

21 Vgl. Badiou A., „Amor y psicoanálisis“, art. cit., S. 106-110. 
Und dann:

\section{$1 \rightarrow 2$; infinitum}

Diese schwierige und lange Kreation der Liebe, die aus einer ereignishaften Begegnung, einer Benennung und dem Weg eines Vorganges besteht, und die die Welt beider Eins durch verschiedene Operationen (eigentlich Lebensentscheidungen) verändert, bezeichnet die Liebe als einen Wahrheitsvorgang.

Nachdem wir uns diesen vier Konzeptionen der Liebe kurz angenähert haben, können wir dann aus einer badiouschen Perspektive das Gedicht von Heine interpretieren.

Unsere These ist, dass dieses Gedicht Heines von einem Aufrichtigkeitsmoment spricht, in dem das lyrische Ich sich an seine Geliebte „Emma“ richtet, um die Entscheidung zu treffen, ob es wirklich zwischen ihnen die ereignishafte Konstruktion von etwas Neuem gibt, oder ob er nur durch seine Begegnung mit ihr die bloße Kontinuität des Gleichen erfahren hat. Und ein solcher Moment fordert eine explizite Bitte um die Wahrheit, wobei die subjektive Schwierigkeit der Entscheidung verursacht, dass die Benennung der Liebe („Emma“) mit dem Ausruf einer Qual („Dilemma“) verschmilzt. Das lyrische Ich fragt sich dann in einem Moment von Ehrlichkeit sich selbst gegenüber, ob all das nur eine fiktionale Narrheit war, oder ob das Reale der Differenz anderswie von beiden erfahren wurde. Deswegen ist dieser Moment, den Heine in Verse bringt, trotz der Qual und der Verwirrung des lyrischen Ich nicht nur ein Moment der Ehrlichkeit, sondern auch ein Moment der Aufrichtigkeit, denn das Gedicht ist das Operatorsymbol davon, dass sowohl dank „Emma“ als auch dank des lyrischen Ich etwas Neues in der Form der Sprache aufgerichtet wurde: das Gedicht selbst. Die Ehrlichkeit des Ich gegenüber seiner Erfahrung der Wahrheit geht über seine eigene Einheit (über seine „Liebestollheit") hinaus, um einen Entscheidungsmoment in der Form eines Sprachkunstwerkes aufzurichten. Diese Poetik der Aufrichtigkeit besteht dann darin, die Entscheidung zu treffen, ob es keine Beziehung und dann nur das Gleiche gibt, oder ob sich die Differenz als Treue zur Bühne der Zwei fortsetzen lässt:

Emma, sage mir die Wahrheit:

Ward ich närrisch durch die Liebe?

Oder ist die Liebe selber

Nur die Folge meiner Narrheit?

Ach! mich quälet, teure Emma, Außer meiner tollen Liebe, 
Außer meiner Liebestollheit,

Obendrein noch dies Dilemma.

Die erste Formalisierung dieses Gedichtes von Heine:

Wahrheit(?) $\rightarrow(($ Liebe $\rightarrow$ Narrheit $) \mathrm{v}($ Narrheit $\rightarrow$ Liebe $))$

kann endlich mit Hilfe Badious nochmals so formalisiert werden:

Wahrheit(?) $\rightarrow((1 \rightarrow 2) \mathrm{v}(1 \rightarrow 1,1))$

\section{Schlussbemerkungen}

In diesem Aufsatz wurde versucht, ein Liebesgedicht von Heinrich Heine mit der Theorie der Liebe von Alain Badiou in Verbindung zu bringen, was als Übung einer Hermeneutik der Liebe bezeichnet werden könnte. Die Hauptthese dieser hermeneutischen Übung war, dass dieses Gedicht als die Symbolisierung eines durch ein Liebesereignis hervorgebrachten Operators der Welt interpretiert werden kann, und dass jene Symbolisierung sich als ein Aufrichtigkeitsmoment ausdeuten lässt, dessen Form, Termini und Ton das Gedicht selbst aufrichtet.

Obzwar bloß sehr knapp gezeigt wurde, dass die Erfahrung der Liebe als ein Wahrheitsvorgang im Rahmen der Philosophie Badious betrachtet werden kann, haben wir uns mit Hilfe des Gedichtes von Heine vornehmlich auf einen Aufrichtigkeitsmoment der Liebe beschränkt. Es wäre sicherlich noch mit Hilfe anderer Werke genauer und ausführlich zu zeigen, wie jene Verbindung zwischen Operator und Welt die Liebe als Wahrheitserfahrung bezeichnet.

Es kann hier aber schon festgehalten werden, dass auf jeden Fall eine solche Wahrheitserfahrung die Verschmelzung oder - um den phänomenologischen Ausdruck zu verwenden - die Verflochtenheit zuvor getrennter Welten zugunsten des Auftauchens eines neuen subjektiven Vorganges bedeuten würde, was Badiou zufolge auch anderen Arten von Wahrheitssubjektivierungen eigen ist, nämlich der Kunst, der Wissenschaft und dem Politischen. Unsere hermeneutische Übung hat aber auch schon implizit gezeigt, dass jene Arten von Wahrheitserfahrungen sich ebenso untereinander zusammensetzen und ineinander verschachtelt sein können, was von Badiou als ihre „composibilité “ bezeichnet wird. Unsere Übung hat versucht, diese Zusammensetzung von Liebe und Kunst im Gedicht Heines $\mathrm{zu}$ fokussieren und $\mathrm{zu}$ formalisieren, um zugleich der Liebeserfahrung und dem 
Kunstwerk eine andere Perspektive und Möglichkeit zu geben. Dies ist Badiou zufolge eine der wichtigsten Aufgaben der Philosophie, nämlich den schon geschehenen Wahrheitserfahrungen andere oder neue Perspektiven, Richtungen und Möglichkeiten zu geben.

Zum Schluss können wir in diesem Sinne noch das Folgende festhalten: obwohl Badiou selbst nicht von Aufrichtigkeit, sondern nur von Entscheidung oder Aussuchen gegenüber dem Ereignis spricht, könnten wir in einer künftigen Untersuchung erforschen, in welchem Sinne auch bei den anderen Arten von Wahrheitssubjekten Aufrichtigkeitsmomente zu finden sind.

In memoriam

Enrique Hülsz Piccone

Óscar Palacios Bustamante (Mexiko-Stadt, 1988) hat Philosophie studiert und kurzzeitig gelehrt. Er hat Lyrik, journalistische und wissenschaftliche Artikel veröffentlicht. Zurzeit promoviert er am Zentrum für Rechtsphilosophie (Centre de Philosophie du Droit, CPDR) der Katholischen Universität von Louvain (Université catholique de Louvain, UCLouvain).

E-mail: noscarpb@gmail.com 